so the unanimity of their histories. All these patients have, but a short time before, recovered from some of those febrile disorders whose sequelæ are so often neglected by parents, and the consequences of which are more fatal than the parent disease. Happily, the expectant timid treatment commonly pursued in the two forms of the same disease already discussed has, in this particular instance, not been followed, owing partly to Sir Astley Cooper's example, and partly, I suspect, to the parts affected being considered less susceptible of injury. I believe that the zinc injection, or one composed of Goulard extract and water, together with iron, and an occasional aperient, is as universally the plan adopted as my experience would lead me to hope for whilst my out-nf-door exercise, and good nourishing diet, will be acknowledged as necessary as in the other forms of this very disagreeable and sometimes dangerous malady.

In tracing the history of these affections of the mucous passages, it has been already said that they are to be found following some exhaustive fever, and very frequently accompanied by the presence of worms in the intestines. This latter circumstance led to the inquiry, if there be any truth in the commonly received opinion, that the irritation produced by the presence of parasites in the intestines was sufficient to account for the discharges mentioned? I conceive that this opinion cannot be held, inasmuch as ulcers are constantly seen in varions parts of the body, produced by poorness of living; and we likewise know that it is only in the intestines of weak children that parasites retain their hold. Both the suppurative discharge and the worms may, and do, exist together, but not as cause and effect; the origin of both must be traced to the atrophied condition of body produced by the wasting fever.

In concluding these few crade remarks upon a class of diseases which has no place or name in any nosological table, and which has been generally looked upon as unworthy of much consideration, I would beg to urge the evil results arising from an a sence of early treatment as a moving cause why we should not overlook things apparently, and only apparently, so trifling. Allow an ulcerated condition of the aural, nasal, and vaginal membranes to continue unchecked, and you will certainly lay the foundation for deafness, loss of the sense of smell, disfigurement of the features, incurable cerebral disorders, and all those distressing diseases to which the internal organs of generation in the female are from so many causes peculiarly obnoxious. One great cause of neglect I believe to have arisen from a fear, lest in treating these discharges from delicate passages in the same manner we do indolent asthenic affections of the body generally, some fancied but undefined ill should arise to the organ itself, or that by suppressing the discharge, neighbouring parts, equally important, should become congested. This fear, I beg to assert, after much careful experience, is not warranted by facts, whilst the magnitude of the evils resulting from non-interference is daily demonstrable.

Lower Brook-street, Grosvenor-square.

\section{REPORT OF A CASE OF ANEURISM OF THE ANTERIOR PALATINE ARTERY.}

BY W. BIRD HERA PATH, M.B. Lond., M.R.C.S. Eng. SURGEON TO ST. PETER'S AND QUEEN ELIZABETH'S HOSPITALS, BRISTOL.

Havrng seen a communication in The LANCET of this week from Dr. Castle of New York, detailing a case of aneurism of the anterior palatine artery, perhaps the Editor will do me the favour to insert in that journal the particulars of a case presenting many points of similarity, which occurred last autumn in my practice.

Master Stone, aged fourteen, called at my surgery, Sept. 15, about middle day, requesting me to examine a swelling in his face, which gave him some inconvenience and pain. The right cheek was swollen over the region of the superior maxilla; upon inspection interiorly, a puffy soft tumour was apparent in the roof of the mouth, about the size of a hazel-nut, red, hot, and apparently an abscess under the palatine membrane. The first molar tooth on that side being loose and somewhat decayed, I, without further examination, decided upon extracting it, and then determined to puncture the abscess. 'The tooth was easily removed, and upon inserting the lancet into the swelling, I was surprised to find but a very small quantity of pus escape, only enough to form a drop, and a slight stain on the lancet. Inmediately after this followed a considerable quantity of blood, certainly a little more fluid than usual, not per saltum, or I should have suspected the cause at once, but it appeared as if excessive hæmorrhage followed the extraction of the tooth, and not from the abscess, as both operations were hurriedly done, in consequence of the pressure of numerous engagements. After some little delay, the hæmorrhage was controlled by cold water, but without any trouble or even the slightest suspicion on my part of the existence of aneurism.

About eleren P.M. on the 16th, I was desired to visit my little patient, who complained of increased pain in the swelling, and headach in consequence of this pain. Upon examination, it was apparent that tumefaction had considerably increased; but not suspecting a pulsating tumour in this position, I did not examine it digitally, except to feel that it was soft and fluctuating. I passed a probe through the former opening-only a little blood followed, but it was not arterial. This having given him relief, he would not suffer any further interference at this time. I prescribed an aperient, to be taken in repeated doses, to lessen the febrile excitement and headach.

In the evening I again saw him, and then gave an opiate and anodyne liniment.

About two o'clock in the morning of the 18 th, I was called up to see him again, as he was suffering very intense pain. Finding the swelling larger than ever, and still thinking it an abscess which had not fully formed itself before, I again punctured it with a lancet, but more towards the centre of the tumour than at first; immediately arterial blood issued in a jet about one-sixteenth of an inch in diameter-per saltum too. My eyes dilated not a little at the spectacle, as my blunder was apparent. I had opened the cavity of a small aneurism in mistake for an abscess. Aneurism in this situation was quite out of my knowledge or suspicion. I had never heard or read of such a case ; blood continued to flow for some minutes in this way, so as to leave no doubt at all of the circumstance. I then controlled the hæmorrhage by pressurethe thumb was sufficient to do it ; I retained the pressure for several minutes, until a coagulum had formed, which was capable of resisting all further hæmorrhage-and I remained with the patient nearly an hour, to prove its efficacy. However, I had not left him long before I was again summoned, as hæmorrhage had occurred in his sleep to considerable extent. Having now provided myself with proper mechanical means of continuing the pressure in my absence, I felt armed to meet the difficulty. A large wine-cork, cut as an arch on the superior surface, shielded with lint, and secured to a narrow bandage, by being sewn to it, was inserted into the roof of the mouth, and fitted tightly to it; the bandage was then carried over the head and again round the crown, sufficiently to secure the whole mechanism in the place intended for it. I did not feel satisfied with this as an effectual mode of controlling future hæmorrhage, but trusted to it as an extempore aide de camp, until daylight should enable me to decide on a better mode of treatment.

Having explained matters to the anxious parents of the little patient, and solicited the assistance of Mr. Nathaniel Smith in the case, it was determined to meet early in the morning.

At nine A.M., 18th September, we met in consultation, and were not a little pleased to find that no further hæmorrhage had occurred-he had kept the compress on nearly three hours ; it had then become loosened, and fallen off. He was cautioned not to touch the swelling in any way, even with his tongue, and he was instructed how to control the hæmorrhage with his thumb should it again occur. No further proceeding in the way of treatment was adopted for the present; but upon carefully examining the swelling digitally, it was ascertained that a largely dilated arterial trunk ran around the internal and anteriorsides of the swelling, though no pulsation could be detected in the external boundary. It was suspected that the anterior palatine artery was the vessel, and that it might be possible to pass an armed needle between the vessel and the palatine surface of the superior maxillary bone, and by tying this ligature around a cork, the obliteration of the artery might be effected. Should this fail, it was agreed to apply the actual cautery - but to proceed no farther without a second consultation.

I am happy to say that the treatment so hurriedly adopted at first was perfectly successful. No further loss of blood occurred; the poor little fellow was much reduced by what had already taken place, and I had to watch him assiduously during many days before I could pronounce him free from all danger of a relapse-the loss of blood produced delirium also to such an extent, that his parents became much alarmed for his future recovery, but this of course gradually passed off, and he slowly attained convalescence. The tumour gradually decreased in size, and at length disappeared altogether; he has no evidence of having ever had such an occurrence at the present time, although ten months have elapsed since the aneurism was first discovered by me.

It is evident, from a review of this case, and the one related by Dr. Castle, that we must use more circumspection in examining tumours in the palatine region. Although we might find thousands of abscesses in the same position, and not again meet with a similar occurrence, yet we might even to-morrow, by accident, have to deplore a similar mistake in diagnosis. 
The case I bave related circumstantially, and without attempting to shield myself from censure; trusting that it will serve as a warning to my brethren not to be too hasty with their lancets-it has taught me a lesson I shall never forget. From the appearance of inflammation, and the pus at the first puncture, it is clear that an abscess existed in connexion with the decayed tooth and the palatine tumour; but the second puncture laid open the dilated and aneurismal trunk of the artery which fed, if not formed, the tumour itself.

Bristol, July 7, 1850.

\section{ON THE DLAGNOSIS OF OVARIAN DROPSY. Bष I. B. BROWN, EsQ., F.R.C.S.,}

CONSULTING-SURGEON TO THE PADDINGTON LYING-IN CAARITY. (Continued from p. 692, vol. i. 1850.)

I wrux now briefly allude to those diseases which may be mistaken for ovarian dropsy, and slightly remark on their peculiar signs. We have-

1st.-Retroflexion and retroversion of the uterus.

2nd.-Tumours of the uterus.

3rd.-Ascites.

4th.-Pregnancy.

5th.-Pregnancy complicated with ovarian dropsy.

6th.-Cystic tumours of the abdomen.

7th.-Distended bladder.

8th.-Accumulation of air in the intestines, especially if

there has been chronic peritonitis leaving some ascitic fluid.

9th.-Enlargement of the solid viscera of the abdomen, the liver, spleen, and kidney.

10th.-Accumulation of fæces in the intestines.

Respecting the first, ovarian dropsy, when it is first detected in the pelvic cavity, between the rectum and vagina, may be confounded with retroversion of the uterus, but a careful examination of that organ will decide the point, for, in retroversion, the os uteri is thrown forcibly forwards and upwards, and the womb is immovable and painful; not so in ovarian dropsy. Retroflexion of the uterus, which has been well described by Dr. Rigby, more elosely resembles ovarian dropsy, but on examination by the uterine sound, and by careful manipulation, the fundus of the uterus can be placed in its natural position.

2nd. -Tumours of the uterus, particularly those with distinct peduncles, may at first be mistaken for ovarian dropsy, but a careful examination, first of the uterus itself, and then of the tumour, with the absence of fluctuation and elasticity, will soon determine the point.

3rd.-Ascites may be, and is, more generally mistaken for this disease. But in ascites the general signs are, swelling of the extremities, and consequent inability to walk; disturbance to digestion, inadequate action of the liver: respiration, and the action of the heart, are all sensibly affected, both because the thoracic organs are themselves sometimes the seat of disease, and also because those organs are compressed upwards by the unusual elevation of the diaphragm. This disease is usually ushered in by irregular or suppressed action of the liver or lidneys, or by chronic peritonitis; the effusion of fluid is general over the abdominal cavity. On placing the patient on one side, the fluid depends to that side, and the sound of the bowels, on percussion, can be heard over the upper side. At the earlier stages, the effusion is seated at the depending portion of the abdomen; on applying pressure firmly over this depending portion, the fluid will be dispersed; and on percussion the sound of the bowels can be heard. The shape of the abdomen is also altered by any position, as upright or horizontal; the fluid, in fact, occupies the cavity of the abdomen from ensiform cartilage to pubes-that is, the upper, middle, and lower thirds of the abdominal cavity. On placing the patient in the upright posture, the fluid descends so as to render the presence of the intestines perceptible in the upper third of the abdomen.

4th.-Pregnancy is sometimes mistaken for ovarian dropsy, and in the first two or three months of utero-gestation, its discrimination often requires great tact and careful examination, for in ovarian dropsy you sometimes have enlarged mammæ, with dark and extended areolæ around the nipple, and also moisture from the nipple itself, with suppression of the menstrual discharge and morning sickness. A case lately came under my notice, where the patient had been pronounced, by more than one medical man, pregnant; but as constitutional derangement increased, and she did not increase in size so fast as was usual in pregnancy, she consulted me; and believing that it was a case of ovarian disease, with that peculiar thick, cheesy matter already referred to, I examined the uterus by the sound, and ascertained that no pregnancy. existed. Subsequent tapping proved the correctness of tho diagnosis. I was also called suddenly to another lady, who had been most carefully examined by a distinguished obstetric physician, (not generally mistaken in his diagnosis,) who declared that she suffered from ovarian dropsy, and yet, on my: arrival, I found her in premature labour of five months' pregnancy, induced probably by the examination she had undergone.

Pregnancy complicated with Ovarian Dropsy.-Now this is perhaps the most difficult of all to distinguish and determine. I have met with three cases of this rare complication. In one I was called to see the lady, pregnant with her second child. I found her generally ill and weak, complaining of the enormous size of her abdomen, and satisfied in her own mind that she should have twins. At the proper period labour came on, and the child was born without difficulty; but on placing $m y$ hand externally, to grasp the uterus, I found I could not feel it, for the pelvic cavity was filled by a soft, elastic tumour, and the. uterus had ascended out of the pelvic cavity, and was abovo this tumour. On introducing $m y$ hand into the vagina, and endeavouring to reach the uterus, to remove the placenta, and pressing my other hand externally over the uterus, I had the pleasure to feel the tumour, which I felt was an ovarian cyst suddenly rupture, and discharge its clear, amber-coloured fluid down the side of my arm. The uterus now descended; the placenta was removed, and a very tight bandage applied. and kept on for several weeks; and on a subsequent confine ment, not a vestige of this tumour could be felt. In the second case, I pronounced my opinion to be, that there was ovarian dropsy, independently of pregnancy. This patient was safely delivered of a full-grown child, and subsequently I tapped the cyst, removed sixteen pints of fluid, and ap. plied tight bandaging. This patient suckled her infant for twelve months. In the third case, the patient was delivered in the country, and came to me directly after her confinement. Tapping and pressure were resorted to successfully.

5 th. Cystic tumours of the abdomen are by far the most difficult to discriminate from ovarian dropsy, and it is only from the earliest history and position that we can draw any probable conclusion; and in the enlarged forms of these tumours it is extremely difficult to diagnose accurately.

6th. Distended bladder may be, and has been, mistaken for ovarian dropsy. A case of this kind came under my notice last year. A young lady, aged twenty-three, came up from the country, stating that she had been under treatment for four months, for "falling down of the uterus," but that during the last month she had become very much enlarged in the body, and her medical attendant thought she was suffering from ovarian dropsy, and therefore she consulted me. I found, on examination, a round smooth tumour, the size of a footal head, rising up from the pubic region, with distinct fluctuation. She stated that she had passed but very little urine for some weeks, and then only in very small quantities at a time. On examination per vaginam, I found a retroverted uterus, the os and cervix pressing firmly up against the neck of the bladder. On replacing the uterus by the uterine sound, and pressing on the tumour through the $a b=$ dominal walls, urine escaped through the urethra; I then introduced a catheter, and drew off seven pints of dark, offen. sive urine, and the tumour disappeared never to return.

7th. Accumulation of air in the intestines, especially if there has been chronic peritonitis, leaving some ascitic fluid. I shall presently illustrate this more fully by relating a case in detail.

8th. Enlargement of the viscera of the abdomen, especially of the liver, of the spleen, or of the kidney. I could illus: trate this subject by mentioning some curious cases of error in diagnosis, in connexion with each of those organs, but I shall merely mention, that in these cases we generally have severe constitutional symptoms pointing out the nature of the disease.

Mr. Harvey related a case of great interest last session at the London Medical Society. It was supposed to be a case of ovarian dropsy; ovariotomy was determined on, but not executed, and when the patient died, the disease was found to be an hydatid cyst, connected with the liver, no ovarian disease whatever existing.

9th.-Accumulation of freces in the intestines is another form of disease not unfrequently mistaken for ovarian dropsy. I once met with a case of simple encysted ovarian dropsy, 